Portland State University

PDXScholar

May 2nd, 11:00 AM - 1:00 PM

\title{
Clustering and Multifacility Location with \\ Constraints via Distance Function Penalty Methods and DC Programming
}

Tuyen Tran

Portland State University

Samuel Reynolds

Portland State University

Thai An Nguyen

Duy Tan University

Mau Nam Nguyen

Portland State University

Follow this and additional works at: https://pdxscholar.library.pdx.edu/studentsymposium

Part of the Physical Sciences and Mathematics Commons

Let us know how access to this document benefits you.

Tran, Tuyen; Reynolds, Samuel; Nguyen, Thai An; and Nguyen, Mau Nam, "Clustering and Multifacility Location with Constraints via Distance Function Penalty Methods and DC Programming" (2018). Student Research Symposium. 16.

https://pdxscholar.library.pdx.edu/studentsymposium/2018/Poster/16

This Poster is brought to you for free and open access. It has been accepted for inclusion in Student Research Symposium by an authorized administrator of PDXScholar. Please contact us if we can make this document more accessible: pdxscholar@pdx.edu. 


\section{METHODS AND DC PROGRAMMING}

Tuyen Tran, Samuel Reynolds, Thai An Nguyen ${ }^{a}$

Faculty Advisor: Dr. Mau Nam Nguyen

Fariborz Maseeh Department of Mathematics and Statistics

PO Box 751, Portland, Oregon 97207

thor was sipported bietnam National Foundation for Science and Technology Development under grant \#101.01-2017.325.

\section{Abstract}

This is a continuation of our effort in using mathematcal optimization involving DC programming in clustering and mutifacility location. We study a penaty method based on distance functions and apply it particularly to a number of problems in clustering and multifacility location in which the centers to be found must lie in some given set constraints. We also provide numerical examples to test our method.

In the current time of "big data", clustering is a very important problem that helps classify data in many fields such as machine learning, pattern recognition, image analysis, data compression, and computer graphics. Given a finite number of data points with a measurement distance, a centroid-based clustering problem seeks a finite number of cluster centers with each data point assigned to the nearest cluster center in a way that a certain measurement distance is minimized.

The main focus here is on solving a number of clustering and multifacility location problems with constraints. We use a penalty method with squared Euclidean distance functions to convert constrained problems to unconstrained problems. Then appropriate DC decompositions and the DCA are used to minimize the penalized objective functions. In the case where the measurement distance is defined by the Euclidean norm instead of the squared Euclidean norm, we use Nesterov's smoothing techniques for reducing the nonsmoothness of the model and for providing a DC decomposition that is favorable for applying the DCA. Our method opens up the possibility of using distance function penalty methods for other problems of DC programming.

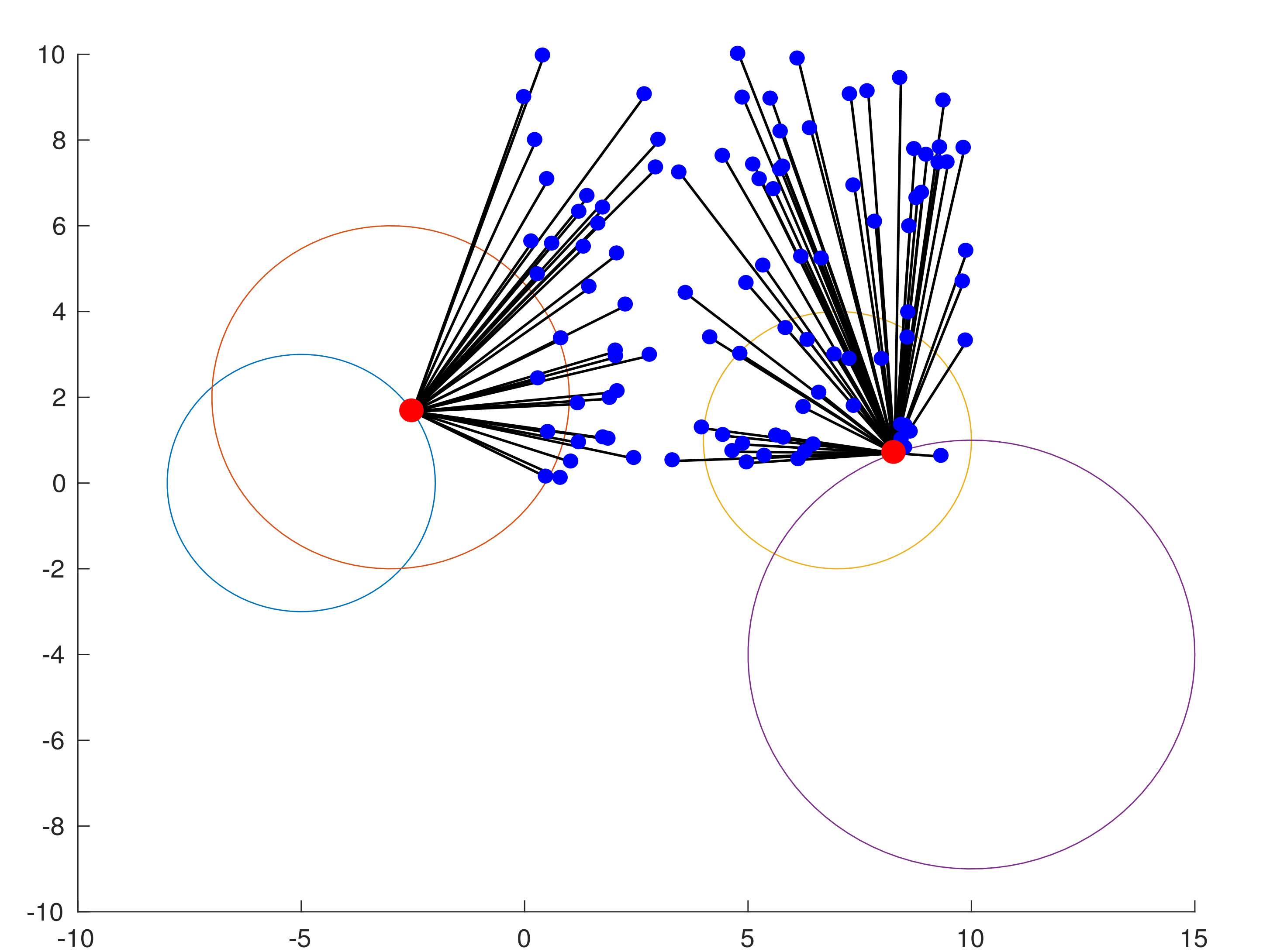

Figure: A clustered data set with $m=100$ random nodes and $k=2$

\section{Clustering with Constraints}

We study problems of clustering with constraints in which the measurement distance is defined by the squared Euclidean norm. We seek $k$ centers $x^{1}, \ldots, x^{k} \in \mathbb{R}^{d}$ of $m$ data nodes $a^{1}, \ldots, a^{m} \in \mathbb{R}^{d}$ and impose the restriction that each $x^{\ell} \in \cap_{i=1}^{q} \Omega_{i}^{\ell}$ for some nonempty closed convex set $\Omega_{i}^{\ell} \subset \mathbb{R}^{d}$ with $\ell=1, \ldots, k$ and $i=1, \ldots, q$. The problem we are concerned with is given by

$$
\begin{array}{ll}
\min & \psi\left(x^{1}, \ldots, x^{k}\right)=\sum_{i=1}^{m} \min _{\ell=1, \ldots, k}\left\|x^{\ell}-a^{i}\right\|^{2} \\
\text { subject to } & x^{\ell} \in \cap_{j=1}^{q} \Omega_{j}^{\ell} \text { for } \ell=1, \ldots, k .
\end{array}
$$

This problem can be converted to an unconstrained minimization problem:

$$
\begin{aligned}
\min & f\left(x^{1}, \ldots, x^{k}\right)=\frac{1}{2} \sum_{i=1}^{m} \min _{\ell=1, \ldots, k}\left\|x^{\ell}-a^{i}\right\|^{2}+\frac{\tau}{2} \sum_{\ell=1}^{k} \sum_{i=1}^{q}\left[d\left(x^{\ell} ; \Omega_{i}^{\ell}\right)\right]^{2}, \\
& x^{1}, \ldots, x^{k} \in \mathbb{R}^{d},
\end{aligned}
$$

where $\tau>0$ is a penalty parameter.

\section{Set Clustering with Constraints}

In this section, we turn our attention to a model of set clustering with constraints, i.e., for given $m$ subsets $\Lambda_{1}, \ldots, \Lambda_{m} \subset$ $\mathbb{R}^{d}$, we seek $k$ cluster centers $x^{\ell} \in \cap_{j=1}^{q} \Omega_{j}^{\ell}$ for $\ell=1, \ldots, k$, where each $\Omega_{j}^{\ell}$ is a subset of $\mathbb{R}^{d}$. The measurement distance is defined by the squared distance functions to the sets involved. The optimization modeling of the problem to be solved is given by

$$
\begin{array}{ll}
\min & \psi\left(x^{1}, \ldots, x^{k}\right)=\sum_{i=1}^{m} \min _{\ell=1, \ldots, k}\left[d\left(x^{\ell} ; \Lambda_{i}\right)\right]^{2} \\
\text { subject to } & x^{\ell} \in \cap_{j=1}^{q} \Omega_{j}^{\ell} \text { for } \ell=1, \ldots, k .
\end{array}
$$

Using the penalty method based on distance functions with a parameter $\tau>0$, we consider the constrained set clustering model:

$$
\min f\left(x^{1}, \ldots, x^{k}\right)=\frac{1}{2} \sum_{i=1}^{m} \min _{\ell=1, \ldots, k}\left[d\left(x^{\ell} ; \Lambda_{i}\right)\right]^{2}+\frac{\tau}{2} \sum_{\ell=1}^{k} \Sigma_{j=1}^{q}\left[d\left(x^{\ell} ; \Omega_{j}^{\ell}\right)\right]^{2},
$$$$
x^{1}, \ldots, x^{k} \in \mathbb{R}^{d}
$$

\section{Multifacility Location with Constraints}

Given a set of $m$ points (nodes) $a^{1}, a^{2}, \ldots, a^{m}$ in $\mathbb{R}^{d}$, our goal is find $k$ centers $x^{\ell}$ for $\ell=1, \ldots, k$, which must be in constraint sets $\cap_{i=1}^{q} \Omega_{i}^{\ell}$ for $l=1, \ldots, k$, such that the transportation cost to the nodes is minimized. The total cost is given

$$
\psi(\mathbf{X})=\psi\left(x^{1}, \ldots, x^{k}\right)=\sum_{i=1}^{m} \min _{\ell=1, \ldots, k}\left\|x^{\ell}-a^{i}\right\| .
$$

We apply Nesterov's smoothing techniques from [4] with smoothing parameter $\mu$ and penalty parameter $\tau$ to approximate the objective function $f_{\tau}$ by a new DC function which is favorable for applying the DCA.

$$
\begin{aligned}
f_{\tau, \mu}\left(x^{1}, \ldots, x^{k}\right) & =\left(\frac{\mu}{2} \sum_{i=1}^{m} \sum_{\ell=1}^{k}\left\|\frac{x^{\ell}-a^{i}}{\mu}\right\|^{2}+\frac{\tau q}{2} \sum_{\ell=1}^{k}\left\|x^{\ell}\right\|^{2}\right) \\
& -\left(\frac{\mu}{2} \sum_{i=1}^{m} \sum_{\ell=1}^{k}\left[d\left(\frac{x^{\ell}-a^{i}}{\mu} ; \mathbb{B}\right)\right]^{2}+\sum_{i=1}^{m} \max _{r=1, \ldots, k} \sum_{\ell=1, \ell \neq r}^{k}\left\|x^{\ell}-a^{i}\right\|+\frac{\tau}{2} \sum_{\ell=1}^{k} \sum_{i=1}^{q} \varphi_{\Omega_{i}^{\ell}}\left(x^{\ell}\right)\right) .
\end{aligned}
$$

References

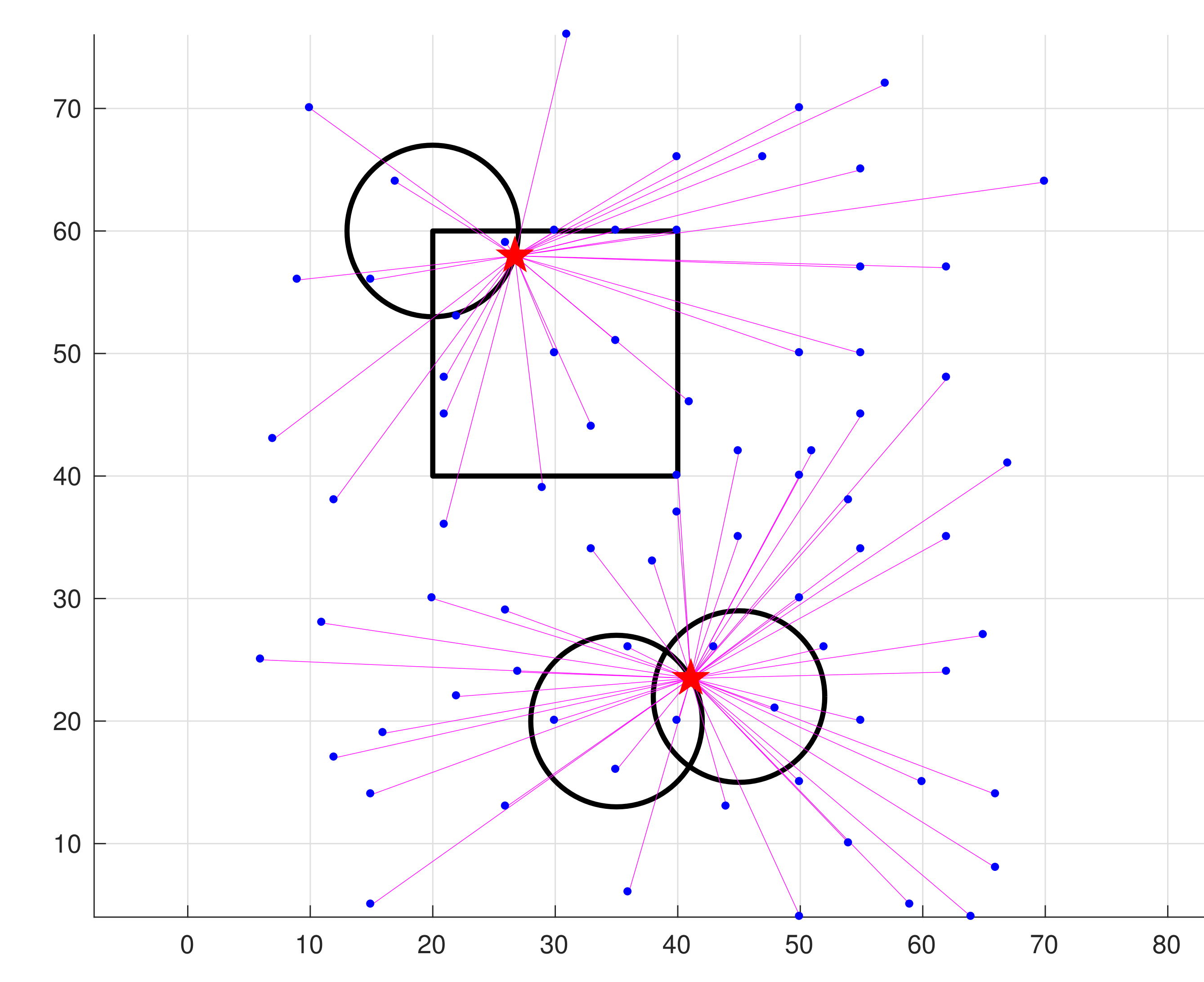

Figure: A 2-center constrained clustering problem for dataset ElL76 taken from the Traveling Salesman Problem Library [3]

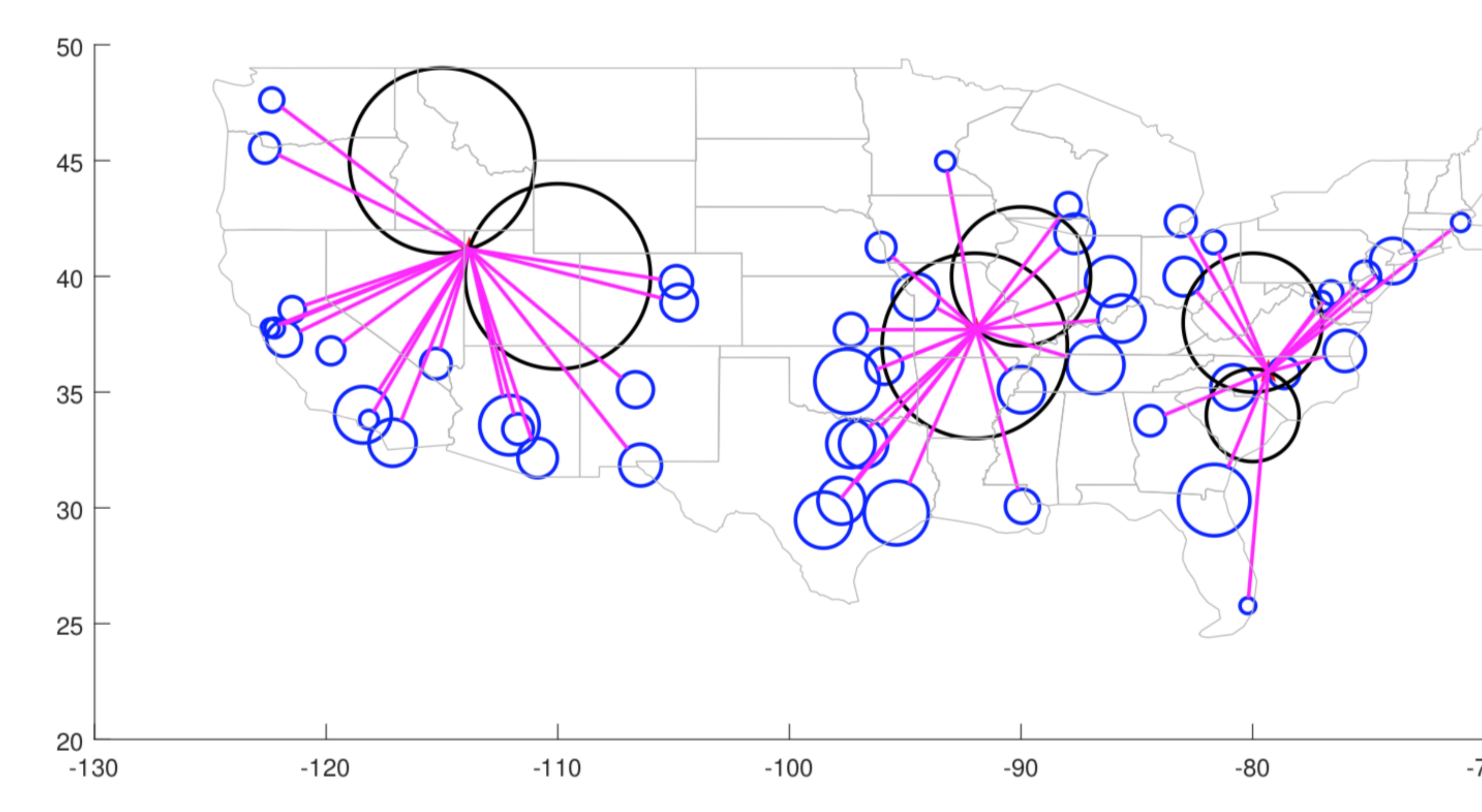

Figure: A 3-center set clustering problems with 50 most populous US cities. Each city is approximated by a ball proportional to its area.

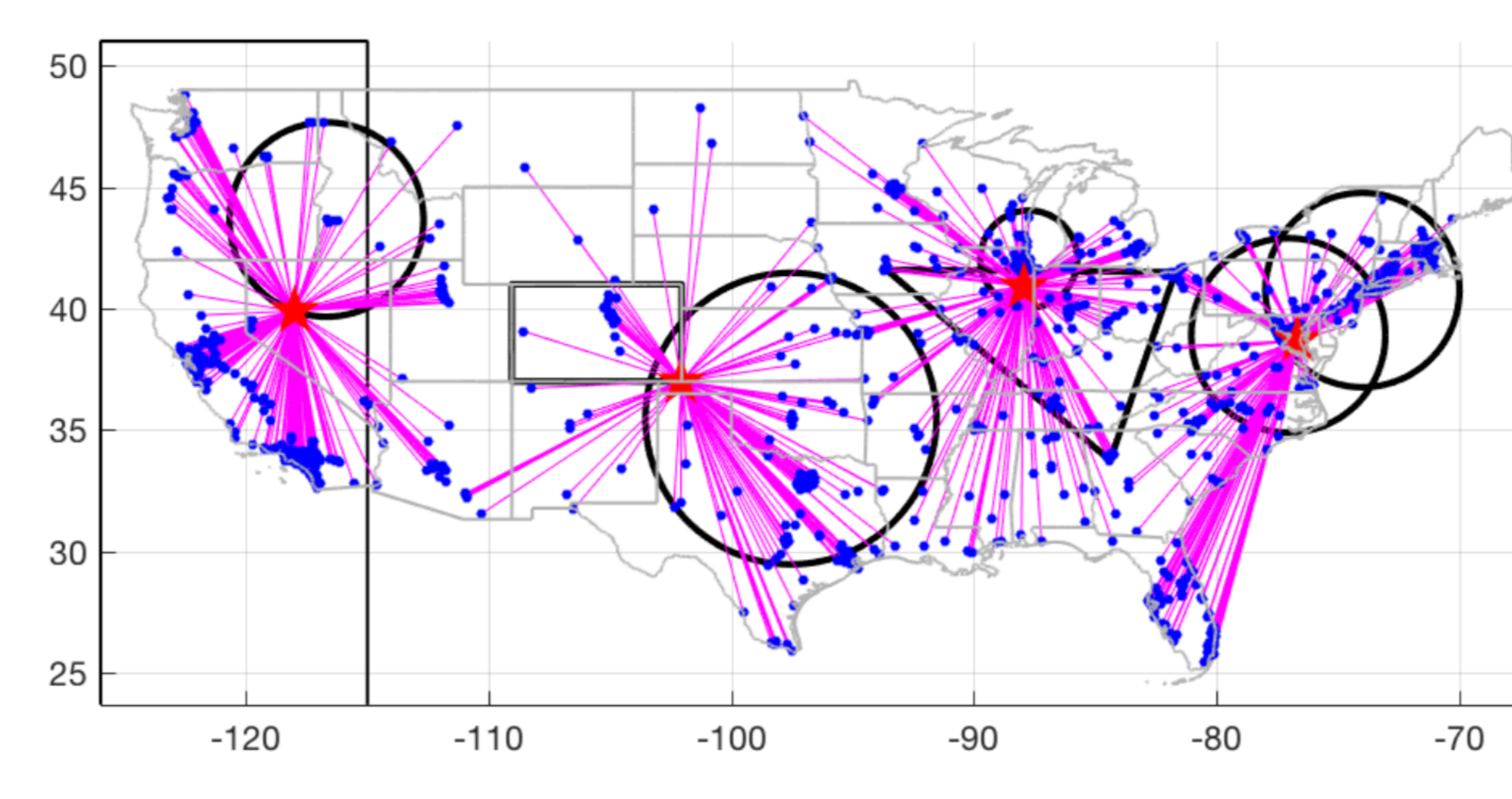

Figure: A 4-center constrained multifacility location problem with US cities dataset. 